Eval uat $i$ on of the effi cacy of the gui del ine on readi ng CT i nages of mal i gnant pl eur al mesot hel i om wi th reference CT fil ms for i mproving the prof i ci ency of radi ol ogi st s

\begin{tabular}{|c|c|}
\hline 著者 & $\begin{array}{l}\text { Zhou Huashi, Tamur a Tar o, Kusaka Yuki nori, } \\
\text { Suganuma Nar uf umi, Subhannachart Pongl ada, } \\
\text { Vi j i t sanguan Chomphunut, Noi si r i heer aya, } \\
\text { Her i ng Kurt G, Aki ra Nasanori, I t oh Har umi, } \\
\text { Ar akawa Hi r oaki, I shi kawa Yui chi, Kumagai } \\
\text { Shi nj i, Kur umat ani Nor i o }\end{array}$ \\
\hline $\begin{array}{l}\text { jour nal or } \\
\text { publ i cat } i \text { on } \mathrm{title}\end{array}$ & EUROPEAN JOURNAL OF RADI OLOGY \\
\hline vol une & 82 \\
\hline nunber & 1 \\
\hline page $r$ ange & 169- 176 \\
\hline year & $2013-01$ \\
\hline URL & ht t p: //hdl . handl e. net /10098/7434 \\
\hline
\end{tabular}




\title{
Evaluation of the efficacy of the guideline on reading CT images of malignant pleural mesothelioma with reference CT films for improving the proficiency of radiologists
}

\author{
Huashi Zhou $^{\text {a,1 }}$, Taro Tamura ${ }^{\mathrm{a}, 2}$, Yukinori Kusaka ${ }^{\mathrm{a}, *}$, Narufumi Suganuma ${ }^{\mathrm{b}, 3}$, \\ Ponglada Subhannachart ${ }^{\mathrm{c}, 4}$, Chomphunut Vijitsanguan $^{\mathrm{c}, 4}$, Weeraya Noisiri $\mathrm{r}^{\mathrm{c}, 4}$, Kurt G. Hering $^{\mathrm{d}, 5}$, \\ Masanori Akira ${ }^{\mathrm{e}, 6}$, Harumi Itoh ${ }^{\mathrm{a}, \mathrm{f}, 7}$, Hiroaki Arakawa ${ }^{\mathrm{g}, 8}$, , Yuichi Ishikawa ${ }^{\mathrm{h}, 9}$, Shinji Kumagai ${ }^{\mathrm{i}}, 10$, \\ Norio Kurumatani $\mathrm{i}^{\mathrm{j}, 11}$ \\ a Department of Environmental Health, School of Medicine University of Fukui, 23-3 Shimoaitsuki, Matsuoka, Eihezi-cho, Fukui Prefecture, 910-1193, Japan \\ ${ }^{\mathrm{b}}$ Department of Environmental Medicine, Kochi University School of Medicine, Japan \\ ' Central Chest Disease Institute of Thailand, 39 Moo 9, Tiwanon Road, Muang Nonthaburi, 11000, Thailand

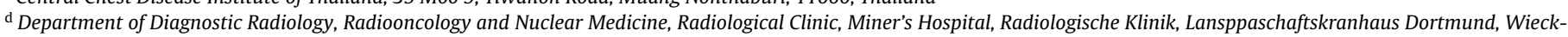 \\ esweg 27, 44309, Dortmund, Germany \\ e Department of Radiology, National Hospital Organization Kinki-Chuo Chest Medical Center, 1180 Nagasone-cho, Kita-ku, Sakai, Osaka, 591-8555, Japan \\ ${ }^{\mathrm{f}}$ Department of Radiology, School of Medicine, University of Fukui, 23-3 Shimoaitsuki Matsuoka, Eiheizi-cho, Fukui Prefecture, 910-1193, Japan \\ g Department of Radiology, Dokkyo University School of Medicine, 880 Oaza-Kitakobayashi, Mibu-cho, Shimotsuga-gun, Tochigi, 321-0207, Japan \\ h Department of Pathology, Cancer Institute, 1-37-1 Kami-ikebukuro, Toshima-ku, Tokyo, 170-8455, Japan

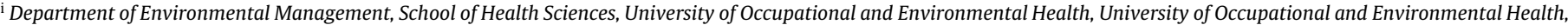 \\ 1-1, Iseigaoka, Yahata-nishi-ku, Fukuoka, Kitakyushu, 807-8555, Japan \\ j Department of Community Health and Epidemiology, Nara Medical University School of Medicine, 840 Shijo-cho Kashihara, Nara, 634-8521, Japan
}

\section{A R T I C L E I N F O}

\section{Article history:}

Received 2 March 2012

Received in revised form 20 May 2012

Accepted 21 May 2012

\section{Keywords:}

Computed tomography

Malignant pleural mesothelioma

Guideline

Sensitivity

Specificity

Agreement

\begin{abstract}
A B S T R A C T
Purpose: To assess the efficacy of the developed guideline on reading CT images of malignant pleural mesothelioma for improving radiologists' reading proficiency.

Materials and Methods: Three radiologists independently read the CT films of 22 cases including definite mesothelioma and non-mesothelioma cases at two times before and after studying the malignant pleural mesothelioma CT Guideline. The sensitivity and specificity for mesothelioma were calculated and compared between the 1 st and 2 nd trials. The kappa statistics was examined for agreement with experts for mesothelioma probability and for mesothelioma features recorded by three radiologists.

Results: After studying the mesothelioma CT Guideline, the sensitivity for mesothelioma shown by the three radiologists at the 2 nd trial was $100 \%, 100 \%$ and $80 \%$, which were higher than $80 \%, 85 \%$ and $60 \%$ at the 1 st trial, respectively. The average kappa for agreement between radiologists and experts on dichotomized mesothelioma probability were 0.69 (good) at the 2nd trial vs. 0.38 (fair) at the 1 st trial. The average kappa for the agreement with experts for each of 7 features by three radiologists were $0.52-0.80$ at the 2 nd trial, which were significantly higher than $0.34-0.58$ at the 1 st trial (Wilcoxon Signed Rank Test: $P<0.01$ ), and as to five features "unilateral pleural effusion", "nodular pleural thickening", "tumoral encasement of lung", "mediastinal pleural thickening", and "diminished lung", they achieved good agreement with average kappa of $0.61-0.80$.

Conclusion: The developed mesothelioma CT Guideline was suggested to have substantial effect in improving the radiologists' proficiency for reading $\mathrm{CT}$ images of mesothelioma, and may contribute to accurate diagnosis of mesothelioma.
\end{abstract}

(ㄷ) 2012 Elsevier Ireland Ltd. All rights reserved.

\footnotetext{
* Corresponding author. Tel.: +81 77661 8335; fax: +81 776618107. E-mail addresses: zhouhua@u-fukui.ac.jp (H. Zhou), tarou@u-fukui.ac.jp (T. Tamura), kusakayk@gmail.com (Y. Kusaka), nsuganuma@kochi-u.ac.jp (N.Suganuma), pongladas@gmail.com(P.Subhannachart),Chompoo_vj@yahoo.com (C. Vijitsanguan), weeraya_tat@yahoo.com (W. Noisiri), k.g.hering@t-online.de (K.G. Hering), akira@kch.hosp.go.jp (M. Akira), hitoh@fmsrsa.fukui-med.ac.jp (H. Itoh), arakawa@dokkyomed.ac.jp (H. Arakawa), ishikawa@jfcr.or.jp (Y. Ishikawa), shkumagai@health.uoeh-u.ac.jp (S. Kumagai), knorio@naramed-u.ac.jp (N. Kurumatani).

${ }^{1}$ Tel.: +81 77661 8338; fax: +81 776618107 .
}
2 Tel.: +81 77661 8335; fax: +81776 618107
3 Tel.: +81 88880 2407; fax: +8188 8802407
4 Tel.: +66 $25803423 \times 7505$; fax: +66 25806992 .
5 Tel.: +49 0231 9221280; fax: +49 02319221648 .
6 Tel.: +8172252 3021; fax: +81722511372.
7 Tel.: +81 77661 8368; fax: +81 776618137 .
8 Tel.: +8128287 2171; fax: +81282864940.
9 Tel.: +8133570 0448; fax: +8133570 0558 .
10 Tel.: +8193691 7282; fax: +81936912694.
11 Tel.: +81 744298841 ; fax: +81 744290673 . 


\section{Introduction}

The International Classification of HRCT for Occupational and Environmental Respiratory Diseases (ICOERD) was developed and used for occupational diseases screening, epidemiology study, and clinical study for respiratory diseases caused by occupational and environmental factors [1]. In order to supply ICOERD, we have developed the Guideline on Reading CT Films of Malignant Pleural Mesothelioma (MPM-CT Guideline) and selected MPM reference CT films [2]. The MPM-CT Guideline provides the terminology of MPM CT features and the MPM probability, the judgment for MPM in terms of involvement distribution and severity, as well as a method to record the CT finding of MPM on the CT reading sheet. The purpose of the current study was to investigate the efficacy of the developed MPM-CT Guideline with the MPM reference CT films for improving the proficiency of the inexperienced radiologists in reading CT images of MPM.

\section{Materials and methods}

\subsection{Subject CT films}

CT films of fifty seven cases including MPM, lung cancer, other malignancies, and benign pleural plaque were collected from the citizens living in the neighborhood of the Kubota factory, a large asbestos cement pipe factory in Amagasaki City, Hyogo Prefecture, Japan. Their MPM was caused by environmental exposure to asbestos (mainly crocidolite) air pollution from the Kubota asbestos factory [3].

Out of the 57 cases, 22 cases including 20 definite MPM cases and 2 pleural plaque cases were subjected to study. The MPM cases were clinically diagnosed as MPM at local hospitals and both pathologically and immunohistochemically confirmed [4,5]. Among the 20 cases of MPM, 7 cases were female, and 13 cases were male. The two cases of pleural plaque were male.

Among the 20 MPM cases, the diffuse type of MPM accounted for $18(90 \%)$, and the localized type for the other 2 cases (10\%). The numbers of the cases with MPM CT features among 20 MPM cases by MPM probability are shown in Table 1 . The CT images of several representative MPM cases among the 20 MPM cases are shown as Figs. 1-5.

\subsection{The MPM reference CT films}

The MPM reference CT films and CT subject images were used by radiologists only at the 2 nd reading trial for comparison of the subjects' CT findings with MPM reference films. The ten typical MPM features on the reference CT films included "unilateral pleural effusion" ("ue"), "nodular pleural thickening" ("nt"), "interlobar fissure thickening" ("it"), "mediastinal pleural thickening” ("mt"), "tumoral encasement of lung" ("te"), "calcified plaque engulfment" ("pe"), "invasion" (iv"), “diminished lung” (“dl”), “contracted hemithorax" ("ch") and "pleural mass" ("pm"). Each MPM feature was indicated by an arrow on the reference CT digital images and hard-copied CT reference films. The CT images of typical MPM features are shown as in Fig. 1 through Fig. 9 in a parallel publication [2].

\subsection{CT reading trials of 22 cases by three radiologists}

Three radiologists participated in independent reading of CT films. All of the radiologists had good proficiency and rich experience in reading CT for pneumoconioses. However, they had not seen many MPM cases previously. Before achieving the 1 st and 2 nd reading trials, they were blinded to the information of the patients' asbestos exposure history, and the clinical and histological diagnosis for any cases.

At the 1 st CT reading trial, the three radiologists were requested to read the monograph of ICOERD and the ICOERD CT reference films, but not to read the MPM-CT Guideline nor the MPM reference CT films, then they read the 22 subject CT films independently. The CT findings associated with asbestosis were recorded according to ICOERD guideline; the MPM features and the MPM probability grade for each case were recorded into the reading sheet according to their experiences.

The interval between the 1 st and the 2 nd CT reading trials by the three radiologists was at least three months. At the 2nd trial, before reading the subject CT films, the three radiologists read the ICOERD guideline with the ICOERD CT reference films again. They also independently studied the MPM-CT Guideline with the MPM reference CT films, and then independently read the 22 subject CT films. They made use of the ICOERD CT reference films and the MPM reference CT films to record the CT findings for pneumoconiosis, the MPM findings, and the MPM probability grade in the CT reading sheets.

\subsection{Statistical analysis}

According to the definition for the MPM probability in the MPMCT Guideline [2], Grade 1 was negative for MPM, no abnormal findings on CT, or the abnormal findings of other diseases; Grade 2 was low probability of MPM; Grade 3 was moderate probability of MPM; Grade 4 was high probability of MPM. Sensitivity for MPM was the proportion of cases for which MPM probability Grade $\geq 2$ recorded by radiologists for each among the 20 MPM cases. Specificity for MPM was the proportion of cases for which MPM probability Grade $=1$ was recorded by individual radiologists among the 2 non-MPM cases, shown as Table A in Supplementary Appendix. The sensitivity and specificity for MPM by the three radiologists were calculated and compared between the 1 st and the 2 nd reading trials.

The weighted kappa for the agreement of the three individual radiologists with the consensus by the four experts (K.G.H., M.A., H. A., H. I.) on the 4-point scale MPM probability was calculated using R-software version 2.14.1 (http://www.r-project.org/), as shown in Table B in Supplementary Appendix. The kappa for the agreement on dichotomized MPM probability was calculated by stratifying the cases with MPM probability Grade 2, 3, 4 into one group, and the cases with MPM probability Grade $=1$ into the other group, as shown in Table $C$ in the Supplementary Appendix. The observed agreement on dichotomized MPM probability between radiologist and experts was also calculated. The calculation of kappa for the agreement on MPM CT feature between radiologist and experts is shown as Table D in the Supplementary Appendix. A kappa value $<0.20$ was considered as poor agreement, $0.21-0.40$ was as fair agreement, $0.41-0.60$ was as moderate agreement, $0.61-0.80$ was as good agreement, and $0.81-1.00$ was as excellent agreement [6]. The kappa values for the 7 MPM CT features by the three radiologists between the 1st trial and 2nd trial were compared by 2-RelatedSamples Nonparametric Test (Wilcoxon Signed Rank Test).

\section{Results}

\subsection{The MPM probability and the 7 MPM CT features among the 20 MPM cases}

The MPM probability and the 7 MPM CT features (7-MPM-CT features) agreed by the four experienced experts for the 20 subject cases of MPM are shown as Table 1. 
Table 1

The number of cases with the 7-MPM-CT features among the 20 MPM cases according to MPM probability.

\begin{tabular}{|c|c|c|c|c|c|c|c|}
\hline \multirow[t]{2}{*}{ Cases with MPM probability } & \multicolumn{7}{|c|}{ The number of cases with the MPM feature } \\
\hline & ue & nt & it & $\mathrm{mt}$ & te & iv & dl \\
\hline Grade $=1(n=0)$ & 0 & 0 & 0 & 0 & 0 & 0 & 0 \\
\hline Grade $=2(n=3)$ & 0 & 1 & 0 & 0 & 0 & 2 & 0 \\
\hline Grade $=3(n=7)$ & 5 & 2 & 4 & 7 & 0 & 1 & 4 \\
\hline Grade $=4(n=10)$ & 8 & 10 & 7 & 10 & 5 & 6 & 10 \\
\hline Total $(n=20)$ & $13(65 \%)$ & $13(65 \%)$ & $11(55 \%)$ & $17(85 \%)$ & $5(25 \%)$ & $9(45 \%)$ & $14(70 \%)$ \\
\hline
\end{tabular}
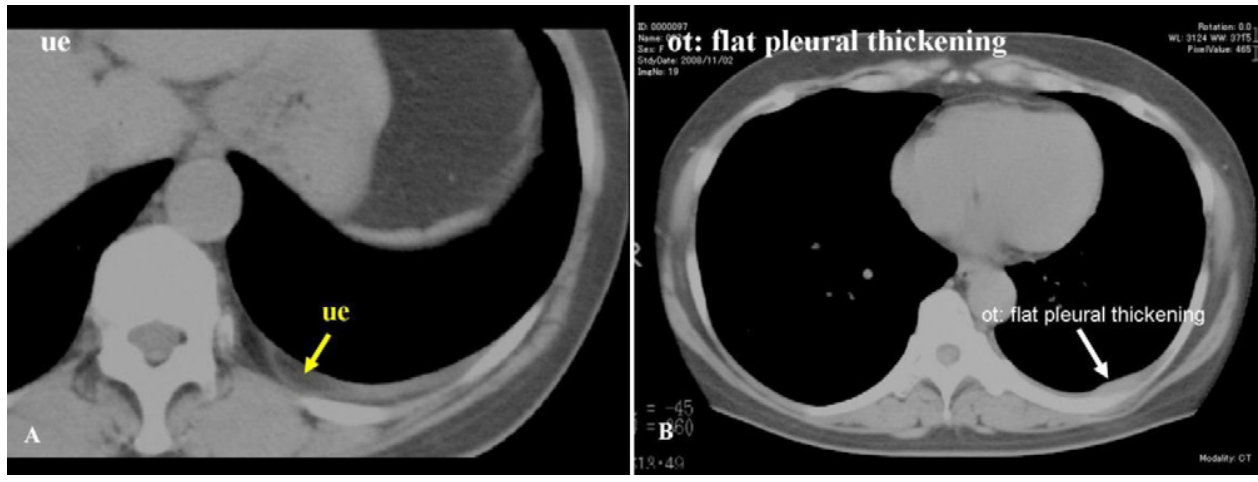

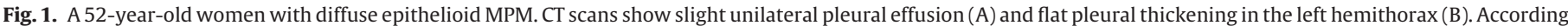
to the CT appearance, the MPM probability was agreed as low probability Grade 2 at mild severity by experts.

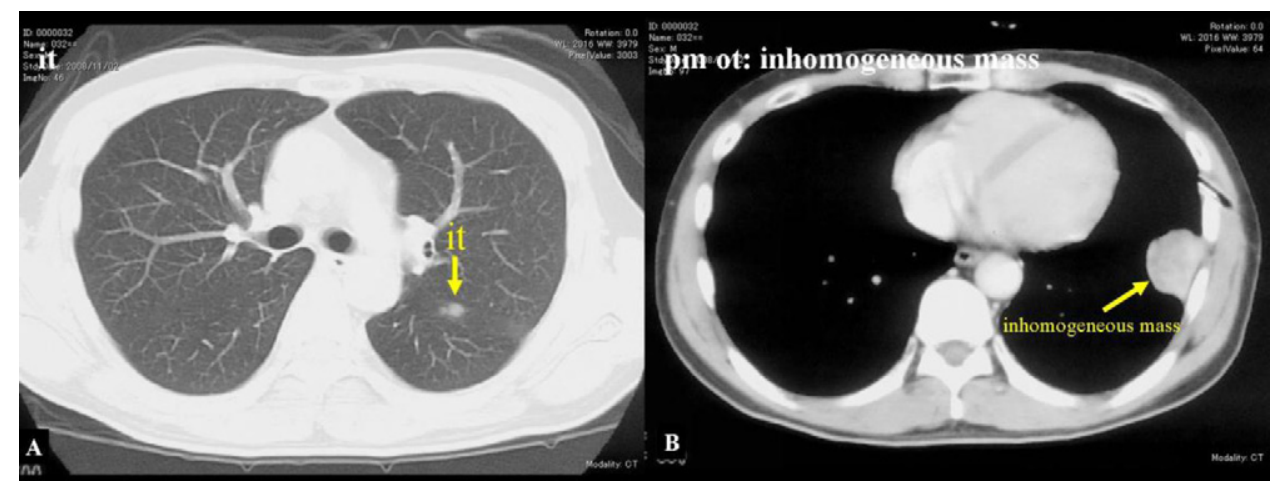

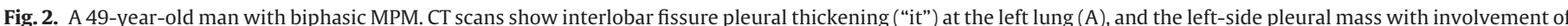

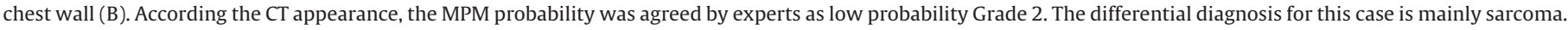

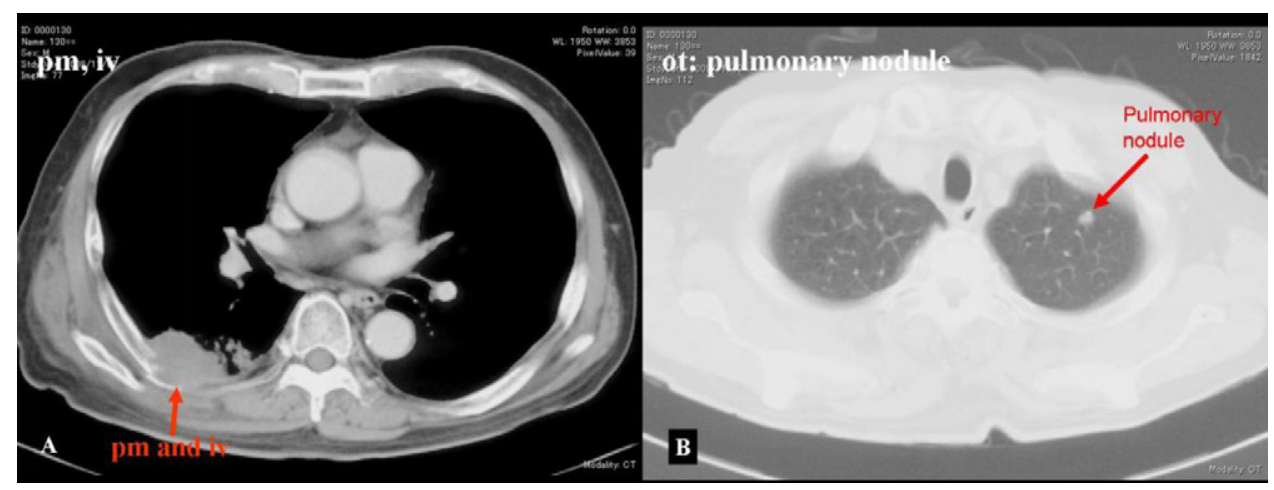

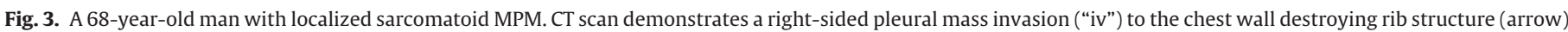

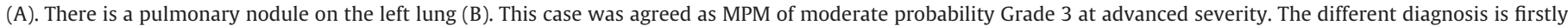
sarcoma and secondly possible metastasis from other cancer.

Please cite this article in press as: Zhou $\mathrm{H}$, et al. Evaluation of the efficacy of the guideline on reading CT images of malignant pleural mesothelioma with reference CT films for improving the proficiency of radiologists. Eur J Radiol (2012), http://dx.doi.org/10.1016/j.ejrad.2012.05.022 

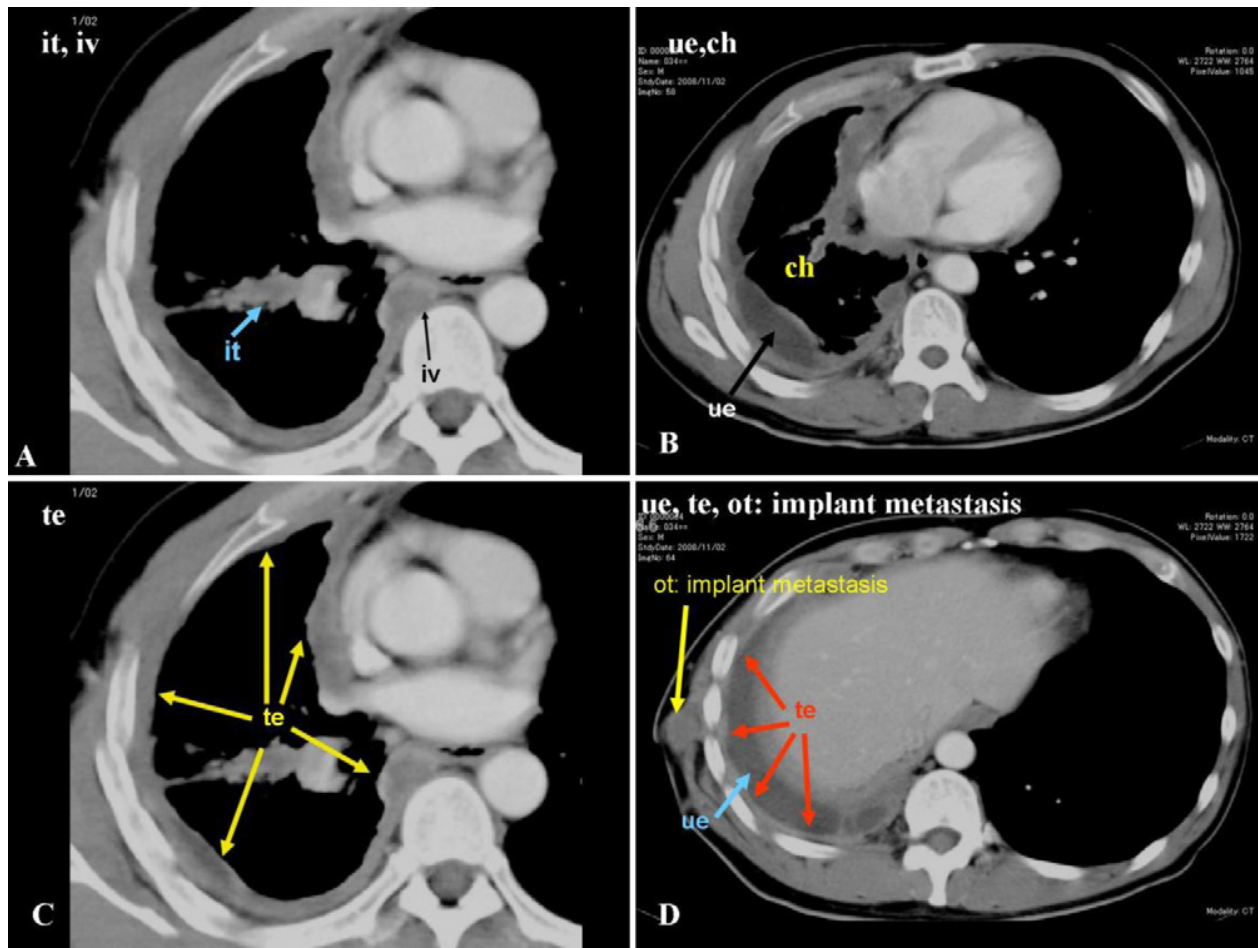

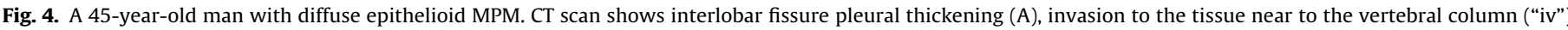

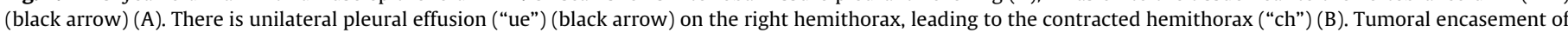

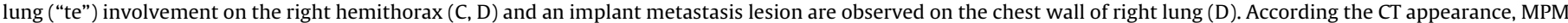
probability was agreed by experts as high probability Grade 4 at advanced severity. This is a typical MPM case.
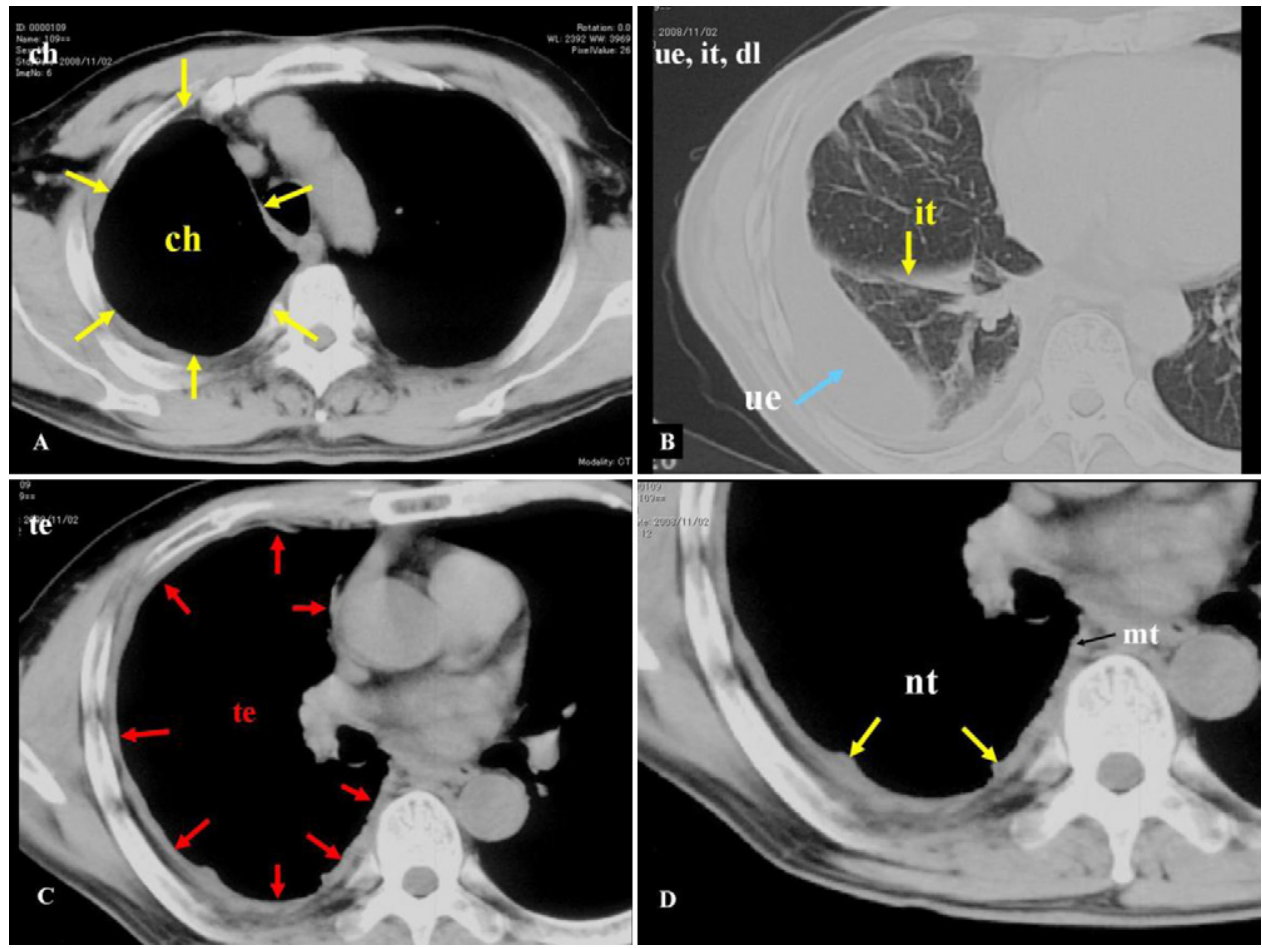

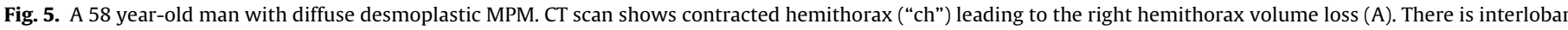

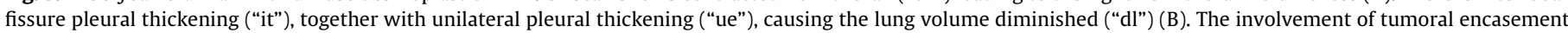

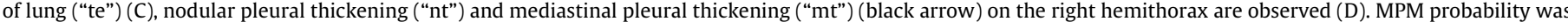
agreed by experts as high MPM probability Grade 4 at advanced severity. This is a typical MPM case. 
Table 2

Agreement on dichotomized MPM probability with 4 experts' consensus and sensitivity and specificity for MPM by three radiologists at the two trials.

\begin{tabular}{|c|c|c|c|c|}
\hline Reader & Reading trial & $\begin{array}{l}\text { Agreement with experts on } \\
\text { dichotomized MPM probability }\end{array}$ & Sensitivity for MPM & Specificity for MPM \\
\hline Radiologist 1 & $\begin{array}{l}1 \mathrm{st} \\
2 \mathrm{nd}\end{array}$ & $\begin{array}{l}18 / 22(81.82 \%) \\
21 / 22(95.45 \%)\end{array}$ & $\begin{array}{l}16 / 20(80 \%) \\
20 / 20(100 \%)\end{array}$ & $\begin{array}{r}2 / 2(100 \%) \\
1 / 2(50 \%)\end{array}$ \\
\hline Radiologist 2 & $\begin{array}{l}1 \text { st } \\
\text { 2nd }\end{array}$ & $\begin{array}{l}19 / 22(86.36 \%) \\
22 / 22(100 \%)\end{array}$ & $\begin{array}{l}17 / 20(85 \%) \\
20 / 20(100 \%)\end{array}$ & $\begin{array}{l}2 / 2(100 \%) \\
2 / 2(100 \%)\end{array}$ \\
\hline Radiologist 3 & $\begin{array}{l}1 \mathrm{st} \\
2 \mathrm{nd}\end{array}$ & $\begin{array}{l}14 / 22(63.64 \%) \\
18 / 22(81.82 \%)\end{array}$ & $\begin{array}{l}12 / 20(60 \%) \\
16 / 20(80 \%)\end{array}$ & $\begin{array}{l}2 / 2(100 \%) \\
2 / 2(100 \%)\end{array}$ \\
\hline
\end{tabular}

Among the 20 definite MPM cases, the cases with MPM probability Grade 2, Grade 3 and Grade 4 accounted for 3/20 (15\%), 7/20 (35\%) and 10/20 (50\%), respectively.

For the 3 cases with MPM probability Grade 2, the cases with the feature "nodular pleural thickening" ("nt") accounted for $1 / 3(33.33 \%)$, and the cases with "invasion" ("iv") accounted for $2 / 3$ (66.67\%).

Among the 7 cases with MPM probability Grade 3, the cases with feature "medialstinal pleural thickening" ("mt") accounted for $7 / 7$ (100\%), the feature of "unilateral pleural effusion" ("ue") were present in 5/7 (71.43\%), "interlobar fissure thickening" ("it") in 4/7 (57.14\%), "diminished lung" ("dl”) in 4/7 (57.14\%), "nt" in 2/7 (28.57\%), and "iv" in 1/7 (14.29\%).

Among the 10 cases with MPM probability Grade 4, the most frequently recorded features were "nt" in 10/10 (100\%), "mt" in $100 \%$, and "dl" in $100 \%$. The cases with feature "ue" were in $80 \%$, "it" in $70 \%$, "iv" in $60 \%$, and "tumoral encasement of lung" in $50 \%$.

\subsection{The sensitivity and specificity for MPM by three radiologists,} the agreement on MPM probability by radiologists with experts

The sensitivity for MPM, specificity for MPM and observed agreement on the dichotomized MPM probability by 3 radiologists at two times of CT readings before and after studying the MPM-CT Guideline are shown as in Table 2.

The sensitivity for MPM by the three radiologists at the 2nd trial was $100 \%, 100 \%$ and $80 \%$, which was higher than $80 \%, 85 \%$ and $60 \%$, respectively at the 1 st reading trial. The observed agreements on dichotomized MPM probability by radiologists were increased at the 2nd trial compared with those at the 1st trial.

The values of weighted kappa for the agreement of MPM probability on the 4-point scale by three radiologists with experts were increased at the 2 nd trial, compared with those at the 1 st trial, as shown in Table 3.

The weighted kappa values for the agreement with experts on 4-point scale MPM probability by three radiologists were $0.32,0.51$ and 0.37 at the 2 nd trial vs $0.24,0.48$ and 0.29 at the 1 st trial, respectively. The kappa value for inter-reader agreements on the dichotomized MPM probability between radiologists and experts at the 2 nd reading trial were 0.65 (good), 1 (excellent) and 0.42 (moderate), respectively, which were significantly higher than 0.42 (moderate), 0.51 (moderate) and 0.21 (fair) at the 1 st trial, respectively. The average kappa for the agreement on dichotomized MPM probability between radiologists and experts were 0.69 (good) at the 2 nd trial vs 0.38 (fair) at the 1 st trial, which seemed to show an upgrading in reading skill.

\subsection{The agreement on MPM features between radiologists and experts}

The results of the agreements for the recorded MPM features between the three radiologists and experts are shown as in Table 4.

For radiologist 1, the agreement with experts for 6 features "unilateral pleural effusion" ("ue"), "nodular pleural thickening” ("nt"), "interlobar fissure thickening"("it"), "mediastinal pleural thickening" ("mt"), "invasion" ("iv") and "diminished lung" ("dl") at the 2nd reading trial was better compared with those at the 1 st trial. The agreement for feature "ue" and "nt" at the 2nd trial was good (kappa $=0.62$ and 0.61 , respectively). The agreement for feature "tumoral encasement of lung" ("te") at the 2nd trial was excellent to the same extent as that at the 1 st trial (kappa $=0.86$ ). The agreement with experts on 3 features ("mt", "iv" and "dl") was markedly increased to excellent (kappa >0.8) at the 2nd trial.

For radiologist 2, the kappa values for the agreement with experts were increased for 5 features ("ue", "nt", "mt", "te" and "dl") at the 2nd trial in comparison with those at the 1st trial. The kappa values for the features "ue", "nt" and "te" showed good agreement with experts (kappa >0.6), and "mt" and "dl" showed excellent agreement (kappa >0.8). The kappa values for the feature "it" and "iv" was 0.55 and 0.51 at the 2 nd trial, which were lower than 0.73 and 0.61 at the 1 st trial, respectively.

For the radiologist 3, the kappa values for 5 features ("nt", "mt", "te", "iv" and "dl") were increased at the 2nd trial in comparison with the 1st trial. The feature "nt" and "te" shows good agreement with expert (kappa $=0.64$ and 0.77 , respectively). The kappa value for the feature "it" at the 2 nd trial was equal to that one at the $1 \mathrm{st}$ trial. The kappa value for the feature "ue" $(0.49)$ at the 2 nd trial was lower than at the 1 st trial (0.65).

The average kappa values of 7 MPM CT features at the 2nd trial by the three radiologists were significantly increased in comparison with the 1st trial, and the six features "ue", "nt", "mt", "te" and "dl" showed good agreement between radiologists and experts (kappa >0.60).

Table 5 shows that the kappa values for the agreement on the 7 MPM CT features by the three radiologists with experts were significantly higher than those at the 1st trial.

Table 3

Agreement on 4-point scale MPM probability and dichotomized MPM probability between radiologist and experts in terms of weighted kappa.

\begin{tabular}{|c|c|c|c|c|}
\hline \multirow[t]{2}{*}{ Reader } & \multicolumn{2}{|c|}{ 4-scale MPM probability $K_{\mathrm{w}}(95 \% \mathrm{CI})$} & \multicolumn{2}{|c|}{ Dichotomized MPM probability $K_{\mathrm{w}}(95 \% \mathrm{CI})$} \\
\hline & 1st trial & 2nd trial & 1st trial & 2nd trial \\
\hline Radiologist 1 & $0.24(-0.06,0.54)$ & $0.32(0.06,0.58)$ & $0.42(-0.09,0.93)$ & $0.65(-0.03,1)$ \\
\hline Radiologist 2 & $0.48(0.19,0.76)$ & $0.51(0.24,0.78)$ & $0.51(-0.01,1)$ & 1 \\
\hline Radiologist 3 & $0.29(0.04,0.53)$ & $0.37(0.12,0.62)$ & $0.21(-0.22,0.65)$ & $0.42(-0.09,0.93)$ \\
\hline Weighted kappa Mean(SD) & $0.34(0.13)$ & $0.40(0.10)$ & $0.38(0.15)$ & $0.69(0.29)$ \\
\hline
\end{tabular}


Table 4

Agreement for the recorded MPM CT features between radiologists and experts by kappa statistics.

\begin{tabular}{|c|c|c|c|c|c|c|c|c|}
\hline \multirow[t]{2}{*}{ Reader } & \multirow[t]{2}{*}{ Reading trial } & \multicolumn{7}{|c|}{ Experts } \\
\hline & & ue & nt & it & $\mathrm{mt}$ & te & iv & $\mathrm{dl}$ \\
\hline \multirow[t]{2}{*}{ Radiologist 1} & $1 \mathrm{st}$ & 0.32 & 0.35 & 0.36 & 0.20 & 0.86 & 0.25 & 0.49 \\
\hline & 2nd & 0.62 & 0.61 & 0.55 & 0.88 & 0.86 & 0.81 & 0.80 \\
\hline \multirow[t]{2}{*}{ Radiologist 2} & $1 \mathrm{st}$ & 0.65 & 0.31 & 0.73 & 0.68 & 0.60 & 0.61 & 0.73 \\
\hline & 2nd & 0.72 & 0.70 & 0.55 & 1 & 0.77 & 0.51 & 0.90 \\
\hline \multirow[t]{2}{*}{ Radiologist 3} & $1 \mathrm{st}$ & 0.65 & 0.37 & 0.45 & 0.39 & 0.28 & 0.25 & 0.17 \\
\hline & 2nd & 0.49 & 0.64 & 0.45 & 0.47 & 0.77 & 0.49 & 0.57 \\
\hline All radiologists & $1 \mathrm{st}$ & 0.54 & 0.34 & 0.51 & 0.42 & 0.58 & 0.37 & 0.46 \\
\hline Mean (SD) & & (0.19) & $(0.03)$ & (0.19) & $(0.24)$ & $(0.29)$ & $(0.21)$ & $(0.28)$ \\
\hline All radiologists & 2nd & 0.61 & 0.65 & 0.52 & 0.78 & 0.80 & 0.60 & 0.76 \\
\hline Mean(SD) & & $(0.12)$ & $(0.05)$ & $(0.06)$ & $(0.28)$ & $(0.05)$ & $(0.18)$ & $(0.17)$ \\
\hline
\end{tabular}

Note: 1 st and 2nd indicates the 1st CT reading trial and the 2nd CT reading trial, $\mathrm{SD}=$ standard deviation of kappa values.

Table 5

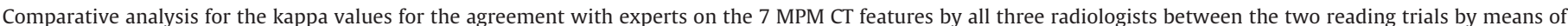
2-Related-Samples Nonparametric Test (Wicoxon Signed Rank Test).

\begin{tabular}{|c|c|c|c|c|}
\hline Карра 2-Карра 1* & Number of pairs & Mean rank & Sum of ranks & $P$ \\
\hline Negative ranks ${ }^{\mathrm{a}}$ & 3 & 4.67 & 14 & $<0.01$ \\
\hline Positive ranks ${ }^{\mathrm{b}}$ & 16 & 11 & 176 & \\
\hline Ties $^{c}$ & 2 & & & \\
\hline Total & 21 & & & \\
\hline
\end{tabular}

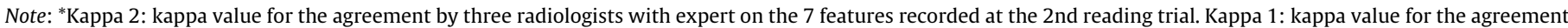
by three radiologists with expert on the 7 features recorded at the 1 st reading trial.

a Kappa $2<$ Kappa 1.

b Kappa $2>$ Kappa 1.

c Kappa 2 = Kappa 1 .

\section{Discussion}

With the development of modern technology, CT scans have now become routine clinical practice for detecting pleural abnormalities in patients. The diagnosis of MPM is usually based on the combination of occupational history, physical and laboratory examination, radiology and the thoracic pathology. CT findings are important to provide the clue in the diagnosis of MPM before any invasive biopsy procedures take place. Diagnosis of MPM at the early stage may enable patients to obtain a better outcome with multiple modality therapy including extensive surgery, chemotherapy, and radiotherapy, which may offer increases in survival time and the life quality for MPM patients [7].

The MPM-CT Guideline was developed by the international experts' efforts [2]. The Guideline provided a standardized way for physicians to record CT findings on subject CT films with the assistance of the MPM reference CT films with typical MPM features. These allow the physicians to make appropriate judgments for the MPM probability, which is determined by the overall impression of the CT findings as a whole and comprehensive evaluation on CT findings being consistent or inconsistent with MPM features, the severity of the diseases, and the distribution of MPM involvement.

Among the 20 definite MPM cases in the current study, the mostly seen features in the number were "mediastinal pleural thickening" ("mt") (85\%), and then were "diminished lung" ("dl") (70\%), the "unilateral pleural effusion" ("ue") (65\%), "nodular pleural thickening" ("nt") (65\%) and "interlobar fissure thickening" ("it") (55\%). The cases with "invasion" accounted for $45 \%$. Most of the 7 features are suggestive of MPM to somewhat through quite extent and frequently common on the CT images of MPM [8].
The relationship between the MPM probability and the number of 7-MPM-CT features in each case was investigated. Among the 20 MPM cases, those cases with the high MPM probability (Grade 3,4 ) were found to have more of 7-MPM-CT features. It was suggested that the more features observed on the CT, the higher MPM probability for the case determined, presenting with a positive correlation. The severity in association with the feature may be the second reason related with probability of MPM. When the features are at severe disease stages, the cases may have high MPM probability, even with only a few features of these.

The relatively high prevalence and frequency of "unilateral pleural effusion" in MPM is of major diagnostic importance [9]. At the early stage of MPM, irrespective of normal-appearing pleura, "unilateral pleural effusion" can be the only finding. The main mechanism of pleural fluid formation in malignancy is lymphatic obstruction. For the cases with pleural effusion and a history of asbestos exposure, MPM should be considered and further investigation should be conducted [10].

There were two features listed in the MPM-CT Guideline "contracted hemithorax" ("ch") and "pleural mass" ("pm") not included in the current comparative analysis, because these two features were later added at the 2nd workshop to the proposed MPM-CT Guideline. The two features are crucial and helpful to make diagnosis of MPM. Solitary masses may occur at the early stage, while multiple masses are more common at later stages [11]. MPM tend to spread along the pleural surface in a "sheet-like" fashion [12]. In CT images with feature "ch", the involved hemithorax is noticeably contracted from a comparison with that in the contralateral lung.

The current study showed that after studying the MPM-CT Guideline, the sensitivity for MPM by all three radiologists was 
increased at the 2 nd trial compared with those at the 1 st trial. For the 1 st radiologist, although the specificity for MPM at the 2nd trial was lower than at the 1 st trial, the observed agreement for the MPM probability was increased to $95.45 \%$ at the 2 nd trial vs $81.82 \%$ at the 1st trial.

Kappa is affected by prevalence of the finding under observation $[13,14]$. In the current study, the proportion of the non-MPM cases among total case was only in $10 \%$, and many cases (50\%) had MPM probability Grade 2 or Grade 3, which had great variances between readers. Therefore the weighted kappa on 4-scale MPM probability proved to be low. However, the analysis with the dichotomized MPM probability showed that two radiologists obtained good and excellent agreements, i.e., kappa $=0.65$ and 1 , respectively. The $3 \mathrm{rd}$ radiologist had achieved moderate agreement with kappa 0.42 on dichotomized MPM probability at the 2 nd trial vs kappa 0.21 at the 1st trial.

The kappa statistics showed that good through to excellent agreements with experts by two of the three radiologists were obtained at the 2nd trial for 5 and 6 features, respectively. For the radiologist 3, there were 5 features including "nt", "mt", "tumoral encasement of lung" ("te"), "invasion" ("iv") and "dl" with increased agreements with experts at the 2nd trial compared with those at the 1 st trial. Compared with those at the 1st trial, the average kappa values of 7 features by the three radiologists were increased at the 2nd trial, in which the five features, i.e., "ue", "nt", "mt", "te" and "dl", showed good agreement with experts. Wicoxon Signed Rank Test showed that the kappa values for the agreement on the 7 MPM CT features between the three radiologists and experts were significantly higher than those at the 1 st trial. These revealed that the radiologists had made improvement in recognition of the MPM CT features at the 2 nd trial compared with the 1 st trial.

Compared to the other features in the 20 MPM cases, the agreement with experts in the feature "invasion" was relatively lower. At the 2 nd trial, the kappa for "invasion" was 0.51 by the 2 nd radiologist and 0.49 by the 3rd radiologist, respectively. This implied that the feature "invasion" was difficult to identify compared to the other MPM features. One reason may be its frequent coexistence with other features that the appearance of this feature may become less notable. The severity of the invasion to the lung structure may be the second reason. If it was less severe, "invasion" may not be easy to identify. This feature of "invasion" is quite suggestive of malignancy. This feature overlaps with metastasis of other carcinoma [15], while the other malignancy also has lung parenchymal involvement.

The average kappa for the agreements between radiologists and experts on feature "interlobar fissure thickening" was 0.51 and 0.52 at the 1 st and 2nd trials, respectively, which were lower than those of other features. The reason may be ascribed to the fact that "interlobar fissure thickening" in some cases is so slight that it may easily be neglected. The feature "interlobar fissure thickening" reflects tumor growth along the interlobar spaces, and may represent one of the earliest significant MPM features, which is seen less frequently in other malignancies or in benign pleural diseases [16].

The CT findings in MPM are not pathognomonic because similar findings may be found in metastatic carcinoma. Nevertheless, they are characteristic. The MPM features can provide valuable information to make a diagnosis of MPM. By identifying the MPM features on CT images, clinicians can recognize MPM [17]. It will be helpful for occupational physicians during the occupational disease screening in health surveillance for workers.

One limitation of the study was that there were only two nonMPM cases with pleural plaque. This may affect the specificity for MPM. Despite the limitation, the results of the comparative study showed that all three radiologists had improved their sensitivity for MPM after studying the MPM-CT Guideline. Two of them had obtained either good or excellent agreements with experts in identifying most of features at the 2 nd trial. These suggested that reliability of the Guideline and the MPM reference CT films to improve the reading proficiency of the radiologists may be validated.

\section{Conclusions}

The current study suggested that the three radiologists improved the proficiency in diagnosis for MPM by identifying of MPM CT features after studying the MPM-CT Guideline with reference MPM CT features. The MPM-CT Guideline and reference CT films may act as good tool to facilitate physicians in recognition of MPM features and contribute to early diagnosis of MPM in the health surveillance.

\section{Conflict of interest statement}

None of the authors has a financial relationship with a commercial entity that has an interest in the subject of this manuscript.

\section{Role of the funding source}

This study was supported by a grant from the Ministry of Education, Sports, Culture, Science and Technology, Japan. The fundholder had no involvement in the study design, collection and analysis and interpretation of data or in the writing of manuscript and in the decision to submit the manuscript for publication.

\section{Acknowledgments}

The author gratefully acknowledge all concerned: Dr. Mitsunori Sakatani, Dr. Seiji Hayashi, Dr. Giichi Inoue, Dr. Masanori Kitaichi, National Hospital Organization Kinki-Chuo Chest Medical Center, Osaka, Japan; Prof. Hisao Shida, Committee of Compensation of Asbestos Related Diseases, Ministry of the Environment Protection, Kawasaki, Japan; Dr. Norihiko Hamada, Department of Radiology, Kochi University, Japan; and we also express thanks to the bereaved family of the patient of MPM and other abnormalities to provide us with the valuable information.

\section{Appendix A. Supplementary data}

Supplementary data associated with this article can be found, in the online version, at http://dx.doi.org/10.1016/ j.ejrad.2012.05.022.

\section{References}

[1] Kusaka Y, Hering KG, Parker JE, editors. International classification of HRCT for occupational and environmental respiratory diseases. Tokyo: Springer-Verlag; 2005.

[2] Zhou H, Tamura T, Kusaka Y, et al. Development of the guideline on reading CT images of malignant pleural mesothelioma and selection of the reference CT films. European Journal of Radiology 2012, http://dx.doi.org/10.1016/j.ejrad.2012.08.008.

[3] Kurumatani N, Kumagai S. Mapping the risk of mesothelioma due to neighborhood asbestos exposure. American Journal of Respiratory and Critical Care Medicine 2008;178:624-9.

[4] Inai K. Pathology of mesothelioma. Environmental Health and Preventive Medicine 2008;13:60-4.

[5] International Mesothelioma Interest Group. A proposed new international TNM staging system for malignant pleural mesothelioma. Chest 1995;108:1122-8.

[6] Suganuma N, Kusaka Y, Hering KG, et al. Reliability of the proposed international classification of high-resolution computed tomography for occupational and environmental disease. Journal of Occupational Health 2009;51:210-22.

[7] Sterman DH, Albelda SM. Advances in the diagnosis, evaluation, and management of malignant pleural mesothelioma. Respirology 2005;10:266-83.

[8] Yamamuro M, Gerbaudo VH, Gill RR, Jacobson FL, Sugarbaker DJ, Hatabu H. Morphologic and functional imaging of malignant pleural mesothelioma. European Journal of Radiology 2007;64:356-66. 
[9] Kawashima A, Libshitz HI. Malignant pleural mesothelioma: CT manifestations in 50 cases. American Journal of Roentgenology 1990;155:965-9.

[10] Fujimoto N, Aoe K, Gemba K, Kato K, Amazaki K, Kishimoto T. Clinical investigation of malignant mesothelioma in Japan. Journal of Cancer Research and Clinical Oncology 2010;136:1755-9.

[11] Yilmaz UM, Utkaner G, Yalniz E, Kumcuoglu Z. Computed tomographic findings of environmental asbestos-related malignant pleural mesothelioma. Respirology 1998;3:33-8.

[12] Miller BH, Rosado-de-Christenson ML, Mason AC, Fleming MV, White CC, Krasna MJ. From the archives of the AFIP malignant pleural mesothelioma: radiologic-pathologic correlation. Radiographics 1996;16: 613-44.
[13] Viera AJ, Garrett JM. Understanding interobserver agreement: the kappa statistic. Family Medicine 2005;37(5):360-3.

[14] Feinstein AR, Cicchetti DV. High agreement but low kappa: I. The problems of two paradoxes. Journal of Clinical Epidemiology 1990;43:543-9.

[15] Leung AN, Müller NL, Miller RR. CT in differential diagnosis of diffuse pleura disease. American Journal of Roentgenology 1990;154:487-92.

[16] Knuuttila A, Kivisaari L, Kivisaari A, Palomäki M, Tervahartiala P, Mattson K. Evaluation of pleural disease using MR and CT with special reference to malignant pleural mesothelioma. Acta Radiologica 2001;42:502-7.

[17] Metintas M, Ucgun I, Elbek O, et al. Computed tomography features in malignant pleural mesothelioma and other commonly seen pleural diseases. European Journal of Radiology 2002;41:1-9. 